\title{
Novel plant transformation system by gene-coated gold particle introduction into specific cell using ArF excimer laser
}

\author{
Shin'ichiro Kajiyama, Fumihide Inoue, Yoshinobu Yoshikawa, Takeshi Shoji, \\ Eiichiro Fukusaki, Akio Kobayashi* \\ Department of Biotechnology, Faculty of Engineering, Osaka University, Suita, Osaka 565-0871, Japan. \\ *E-mail: kobayashi@bio.eng.osaka-u.ac.jp Tel\&Fax: +81-6-6879-7423
}

Received December 27, 2006; accepted February 23, 2007 (Edited by K. Yoshida)

\begin{abstract}
Novel methods for foreign-gene introduction into specific cells are required for the functional analysis of specific cells. We report a new transfection method for intact plant cells that uses ArF excimer laser-induced shock waves to introduce foreign gene-coated gold particles into plant cells through the cell wall. Foreign gene $(s G F P)$-coated gold particles were randomly scattered on the surface of Torenia tissue samples; the laser was irradiated toward the gold particles using a hollow optical fiber and a quartz light-condensing tip to introduce the particles into the target cells. Treated plant tissue samples were then cultured for several weeks on the selection medium, and transformation was assessed by fluorescence microscopy and PCR. Irradiation-induced damage to the cells and coated DNA was insignificant, and the transformation efficiency was similar to that of biolistic transformation based on the particle number. Although this method requires a suitable laser setup and is time-consuming, it could be applied in transformation experiments on specific target cells. Moreover, this method can possibly be used to introduce not only DNA but also biologically active substances such as protein regulators or inhibitors, whose introduction into cells is difficult through the cell wall.
\end{abstract}

Key words: ArF excimer laser, cell-specific transformation, gold particle, laser irradiation.

With the progress of biotechnology, novel plant-cell manipulation methods have been sought, particularly for foreign-gene introduction, because such novel methods are necessary for obtaining stable and/or transient transformants (Bock 2001). Several methods are currently available for introducing exogenous DNA into plant cells (Rakoczy-Trojanowska 2002). A wide range of dicotyledonous and monocotyledonous plant species have been transformed by PEG-mediated direct DNA uptake, electroporation, and fusion of protoplasts with liposomes (Prescott et al. 1998). However, these methods require protoplast formation and efficient cell-wall regeneration systems (Neuhaus and Spangenberg 1990). Agrobacterium-mediated (Hohn et al. 1989) and biolistic transformations (Daniell 1997) have routinely been used in many experiments for tissue explants and cells with intact cell walls. However, these methods also have their own drawbacks. In the former, the transformation efficiency largely depends on the susceptibility of the host plant; in the latter, the risk of tissue damage cannot be avoided because this method uses gunpowder or highpressure gas for particle introduction. In addition, these 2 methods transform the cells in the tissue randomly and are not applicable to cell-specific transformation in principle (Neuhaus and Spangenberg 1990; Russell et al. 1993).

In the previous paper, we reported a novel plant cell manipulation technique using ArF excimer laser (Kajiyama et al. 2006). Although this technique enabled cell specific gene introduction into intact plant cell, the damage of the target cell was not avoidable because it required the insertion of glass capillary through the cell wall aperture made by the laser irradiation. Here we describe an alternative method for transfecting foreign materials into intact plant cells. This method uses ArF excimer laser-induced shock waves to introduce foreign DNA-coated gold particles into the cells. It has 2 major advantages: first, the robust delivery of exogenous DNA only into the target cell and second, minimal damage to the tissue samples.

\section{Materials and methods}

Plant material, tissue preparation, and regeneration conditions

The plant material was prepared from Torenia hybrida cv.

Abbreviations: CaMV, cauliflower mosaic virus; EtBr, ethidium bromide; FDA, fluorescein diacetate; HPT, hygromycin phosphotransferase; RT-PCR, reverse transcriptase polymerase chain reaction; sGFP, synthetic green fluorescent protein; TE, trishydroxymethylaminomethane-ethylenediaminetetraacetic acid

This article can be found at http://www.jspcmb.jp/ 
Summerwave Blue (Suntory Flowers, Japan), an herbaceous plant that can regenerate from apical and stem epidermal cells (Tanimoto and Harada 1984). The Torenia plants (4-6 weeks old) were grown in a growth chamber (Model: MLR$350 \mathrm{H}$; Sanyo Electric, Japan) at $25^{\circ} \mathrm{C}$ under a 16 -h light $\left(120 \mu \mathrm{E} \mathrm{m}^{-2} \mathrm{~s}^{-1}\right) / 8$-h dark cycle. Shoot apices and stem segments were used for gene introduction. The shoot apices were excised under a stereoscopic microscope (Model: SZ60; Olympus, Japan) by using a scalpel and a stainless-steel needle. The stem segments (length, $2-5 \mathrm{~mm}$ ) were prepared by cutting the second node of mature Torenia plants. They were then pretreated in regeneration medium (Murashige and Skoog medium containing $1 \mathrm{mg}^{-1}$ 6-benzylaminopurine, 3\% (w/v) sucrose, and 3\% (w/v) gellan gum; $\mathrm{pH} 5.8)$ for 2 days prior to exogenous gene introduction.

\section{Laser setup}

The laser-irradiation setup used in this study is illustrated in Figure 1. It consisted of a laser oscillator (Model: ArF Excistar S-200; Tuilaser Inc., Germany), a microscope (Model: Ecrips ME600; Nikon, Japan) with a super-long working distance objective lens (Model: CFI Plan EPI SLWD 50X; Nikon, Japan), and a micromanipulator (Model: 5171; Eppendorf Inc., Germany). The laser beam (wavelength: $193 \mathrm{~nm}$, pulse duration: $10 \mathrm{~ns}$ ) was introduced into a hollow optical fiber (Matsuura and Miyagi 1999) (OD: $680 \mu \mathrm{m}$, ID: $500 \mu \mathrm{m}$; Soken, Japan) by using a converging lens, and it was condensed at the end of the fiber by using aluminum-coated quartz tips. The condensing quartz tips were prepared from quartz glass capillaries (Model: Q120-90-10; Sutter Inc., USA) by using a $\mathrm{CO}_{2}$ laser puller (Model: P-2000; Sutter Inc., USA) with the following parameters: heat, 820; fil, 4; vel, 50; del, 130 and the outer surface of the tips were coated with aluminum by using vacuum deposition equipment (Model: VPC-260; Ulvac, Japan). The hollow fiber and laser-condensing tip were attached to the micromanipulator, and the laser could be focused onto any part of the sample surface. The laser energy from the tip was monitored using a power meter (Model: NOVA; Ophir Optronics Ltd., Israel) with a PD-10 or PE-10 counter head (Ophir Optronics Ltd., Israel). Before the laser irradiation toward the plant sample, the periphery of the sample stage was sterilized by $70 \%$ ethanol aq. and UV irradiation $(10 \mathrm{~W}$ for

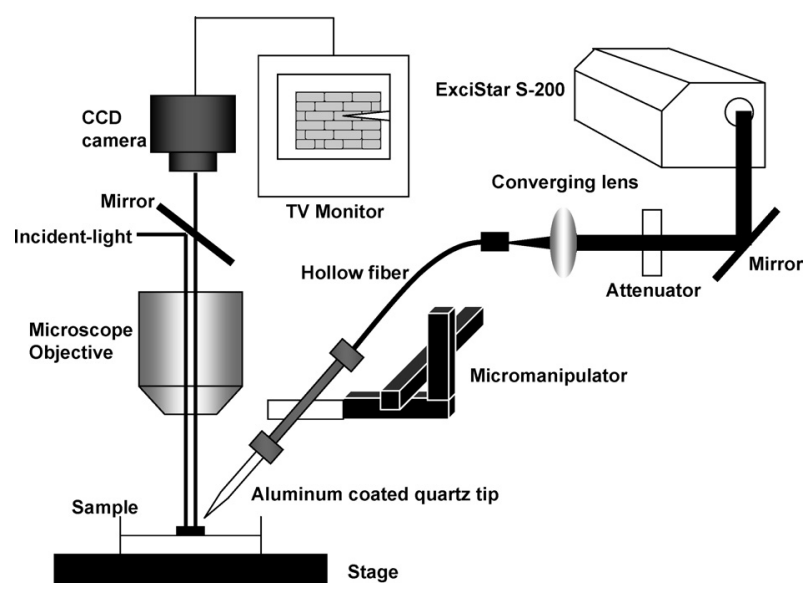

Figure 1. Excimer laser microirradiation setup.
$30 \mathrm{~min}$ ) and gene introduction procedure was performed under the aseptic condition.

\section{Foreign-gene (plasmid) preparation}

The DNA of the pBIsGFP (S65T) plasmid was used as the foreign DNA in this experiment, and it was supplied by Dr. Norihiro Mitsukawa (Toyota Central R\&D Labs., Japan). This plasmid comprised CaMV 35S promoter-driven sGFP (S65T) (Niwa et al. 1999), HPT, and the NOS terminator. The plasmid was prepared using a plasmid maxiprep kit (Bio-Rad, USA) according to manufacturer's instructions. After assessing the purity by $1 \%$ agarose gel electrophoresis, the plasmid DNA was coated onto gold particles ( $2 \mu \mathrm{g} \mathrm{DNA} / \mathrm{mg}$ gold particles) (1- $\mu \mathrm{m}$ gold particles for bombardment, Bio-Rad, USA) according to the Helios Gene Gun instruction manual (BioRad, USA). The gene-coated particles were refrigerated at $4^{\circ} \mathrm{C}$ until use.

\section{Gene-introduction procedure}

Each plant sample, i.e., the pretreated shoot apices and stem segments, was placed on a $0.8 \%(\mathrm{w} / \mathrm{v})$ agar plate. The foreign DNA-coated gold particles were then randomly scattered on the surface of the tissue samples by using stainless-steel needles. Further, the laser was irradiated toward the gene coated-gold particles with an energy of $3 \mathrm{~nJ} /$ shot (energy density: $30 \mathrm{~mJ} / \mathrm{cm}^{2}$, irradiation area: $10 \mu \mathrm{m}^{2}$ ), using the laser setup (Figure 2). The irradiation area was set to approximately $10 \mu \mathrm{m}^{2}$ by controlling the distance between the sample and the end of the quartz tip with the micromanipulator. The number of particles introduced into a single cell was $1-5 /$ shot. The transfected tissue samples were then transplanted onto the selection medium (Murashige and Skoog medium containing $1 \mathrm{mgl}^{-1}$ 6-benzylaminopurine, $30 \mathrm{mg}^{-1}$ hygromycin, $3 \%(\mathrm{w} / \mathrm{v})$ sucrose, and $3 \%(\mathrm{w} / \mathrm{v})$ gellan gum; $\mathrm{pH} 5.8$ ) and cultured in the growth chamber at $25^{\circ} \mathrm{C}$ under a 16-h light $\left(120 \mu \mathrm{E} \mathrm{m}^{-2} \mathrm{~s}^{-1}\right) / 8$-h dark cycle. Nonirradiated samples and samples introduced with gene-uncoated gold particles were separately prepared and used as controls.

\section{Cell viability test}

Twenty-four hours after laser irradiation $(1,3,10$, or $30 \mathrm{~nJ} /$ shot), 100 Torenia stem epidermal cells were vital-stained with FDA (Marfori et al. 2003), and the viable and nonviable cells were counted under a fluorescence microscope (Model: BX-50 with a U-MWB filter set; Olympus, Japan). The tests were repeated 5 times independently and the mean and standard deviation were calculated.

\section{Estimation of DNA damage upon laser irradiation}

The $s G F P(S 65 T)$ plasmid-coated gold particles $(100 \mu \mathrm{g})$ were placed on a $1 \times 1 \mathrm{~cm}$ parafilm ${ }^{\circledR}$ square and irradiated with the ArF excimer laser at the energy density of $40 \mathrm{~mJ} / \mathrm{cm}^{2}$ which is ca. $30 \%$ higher than the energy density used for particle introduction experiments. DNA was extracted from both the laser irradiated and nonirradiated particles by using TE buffer. The extracts were quantified by the comparative $\mathrm{Ct}$ method, using the GeneAmp PCR system (Model: 9700; Applied Biosystems) with the forward primer 5'-GGAGCGCACCATCTTCTT-3', reverse primer 5'- ATGCCCTTCAGCTCGAT-3', and the TaqMan probe 5'-AGACCCGCGCCGA-3'. A standard 
curve was plotted using a known amount of the DNA sample (data not shown). The thermal cycling conditions were $50^{\circ} \mathrm{C}$ for $2 \mathrm{~min}$ and $95^{\circ} \mathrm{C}$ for $15 \mathrm{~min}$, followed by 40 cycles of $94^{\circ} \mathrm{C}$ for $15 \mathrm{~s}$ and $60^{\circ} \mathrm{C}$ for $1 \mathrm{~min}$. The test was repeated 5 times independently, and the amount of DNA in each sample was calculated by comparing it with the standard curve. Data are presented as means and standard deviations.

\section{PCR for transfected genes}

The genomic DNA of the samples, including the adventitious shoots that were generated 6-8 weeks posttransfection, was extracted and purified using the DNeasy Plant Mini Kit (Qiagen, Japan). The DNA was checked for the presence of foreign genes by PCR with the primers 5 '-CATCTGGTGGTGTGAGCAAG-3' and 5'-ATGCCGTTCTTCTGCTTGTC-3' for $s G F P$ and $5^{\prime}$-GAATTCAGCGAGAGCCTGAC-3' and 5'-ACATTGTTGGAGCCGAAATC-3' for HPT. The thermal cycling conditions were $94^{\circ} \mathrm{C}$ for $5 \mathrm{~min}$, followed by 40 cycles of $94^{\circ} \mathrm{C}$ for $1 \mathrm{~min}, 60^{\circ} \mathrm{C}$ for $1 \mathrm{~min}$, and $72^{\circ} \mathrm{C}$ for $1 \mathrm{~min}$. The PCR products were separated by $1 \%$ agarose gel electrophoresis and visualized by $\mathrm{EtBr}$ staining. The transformation efficiency was calculated as the percentage of trials with transgene-expressed tissues (from a total of 279 trials for stem epidermis and 277 trials for shoot apex samples).

\section{Fluorescence microscope observation}

The tissue samples were checked for $s G F P$ expression at $24 \mathrm{~h}$, $72 \mathrm{~h}$, and 1 week after gene introduction by using a fluorescence microscope (Model: BX50 with a U-GFP-A mirror set; Olympus, Japan), and micrographs were obtained using a CCD digital camera system (Model: DP-70; Olympus, Japan). The samples that survived for a week after selection were monitored once a week using the same equipment.

\section{Results and discussion}

\section{Effect of the laser irradiation on Torenia epidermal cells}

The viability of the laser-treated cells was determined to estimate the extent of irradiation-induced damage. Laserinduced shock waves can be produced when a pulse laser is focused on a solid target (Yoshida 2004). In our experiment, using an aluminum-coated light condensing

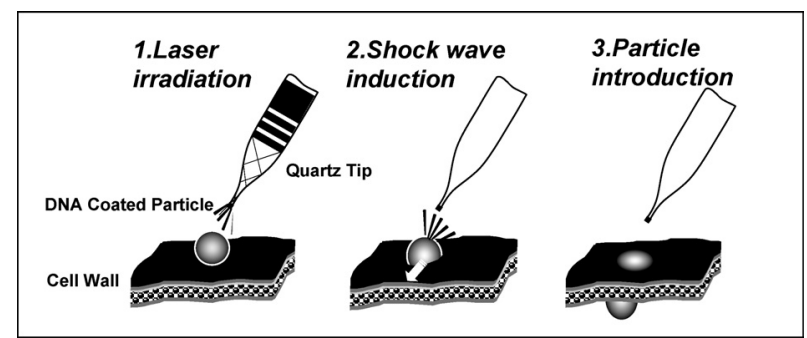

Figure 2. Schematic diagram of particle introduction by laser irradiation. The laser was irradiated through laser condensing quartz tip toward the DNA coated gold particle (1). Upon laser irradiation, the shock wave was induced on the surface of the particle and this generated the impelling force of the particle to smash into the cell inside $(2,3)$. tip, an ArF excimer laser (wavelength: $193 \mathrm{~nm}$, pulse duration: $10 \mathrm{~ns}$ ) was focused on gene-coated gold particles placed on the surface of plant cells, and the particles were thus successfully introduced into the plant cells with intact cell walls (Figure 2). ArF excimer lasers have very low permeability, and the laser pulse should not damage important biological molecules within the cells. At an energy of $3 \mathrm{~nJ} / \mathrm{shot}$, which was sufficient for particle delivery into the cell, approximately $90 \%$ cell viability was retained (Figure 3). However, when the laser energy was increased to $30 \mathrm{~nJ} / \mathrm{shot}$, the cell wall

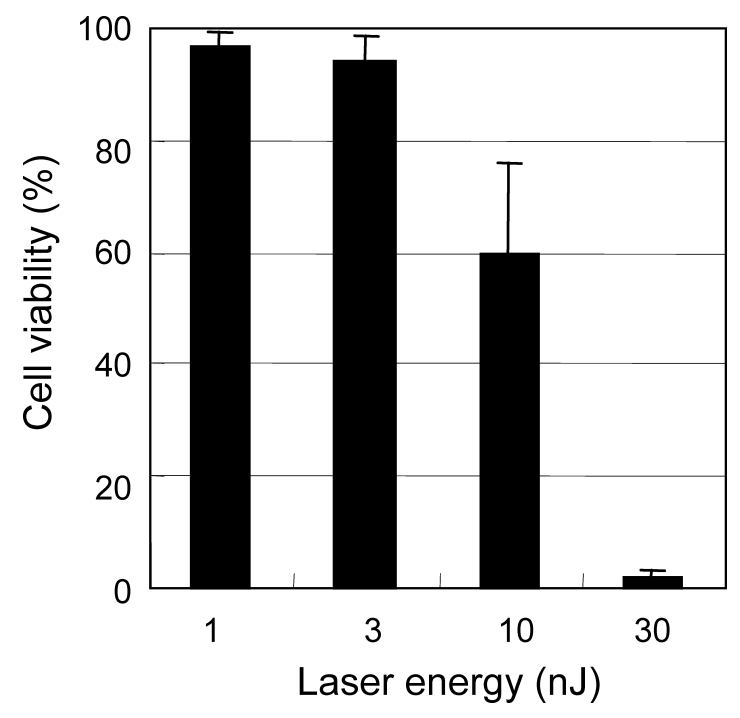

Figure 3. Cell viability of laser-treated Torenia epidermal cells $24 \mathrm{~h}$ after irradiation. One hundred cells were irradiated at various energies and vital stained with FDA. The values are the percentages of viable cells \pm SD

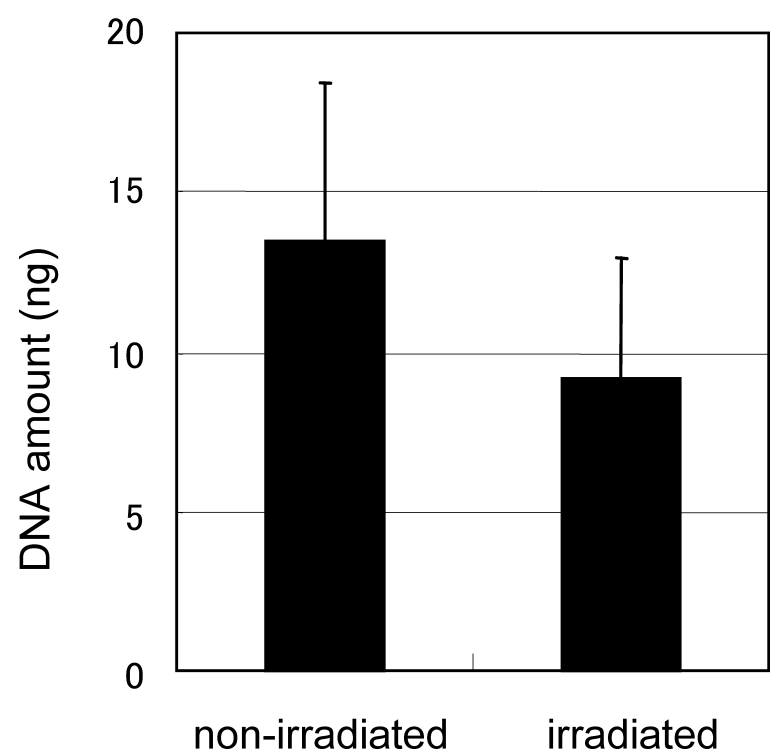

Figure 4. The effect of laser irradiation on DNA. The plasmid pBIsGFP (S65T) was independently extracted from both the laserirradiated and nonirradiated particles by using TE buffer and quantified by quantitative real-time PCR. The test was repeated 5 times independently. Data are presented as means and standard deviations. 
was partially perforated and part of the cytoplasm was observed to be extruded. At an energy of $1 \mathrm{~nJ} / \mathrm{shot}$, the particles did not penetrate the cells.

\section{DNA damage upon laser irradiation}

The diminution of the coated DNA was also evaluated by quantitative real-time PCR, in case the laser had caused serious DNA damage. Figure 4 shows the amount of DNA recovered from the laser-irradiated and nonirradiated particles. Although approximately 30\% of the DNA was depleted by irradiation with the ArF excimer laser at the energy density of $40 \mathrm{~mJ} / \mathrm{cm}^{2}$ ( $c a$. $30 \%$ higher laser density than that used in particle introduction experiment), over $10^{2}$ copies of DNA per
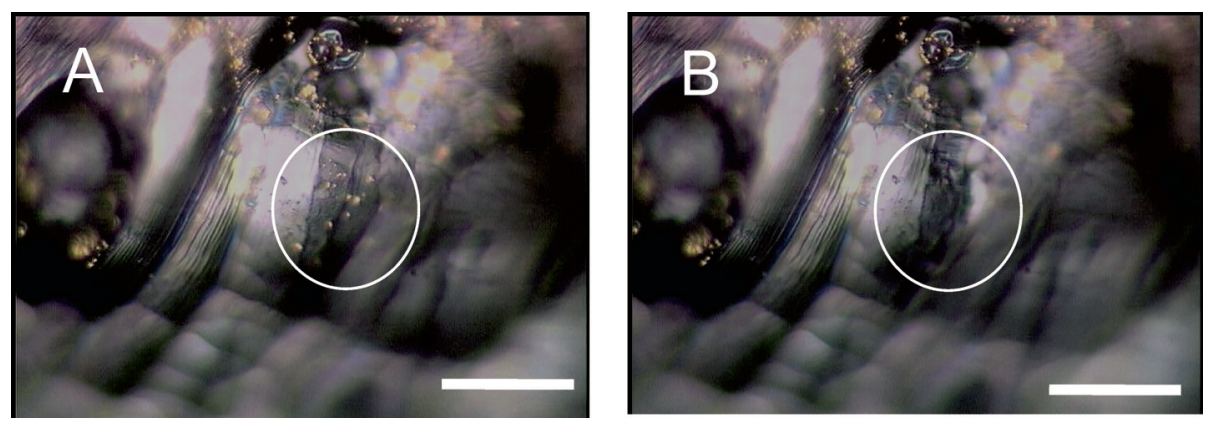

Figure 5. Micrographs of laser-treated samples. Torenia epidermal cells prior to (A) and after laser irradiation (B). The circles indicate the targeted gold particles. (Scale bar: $50 \mu \mathrm{m}$ )
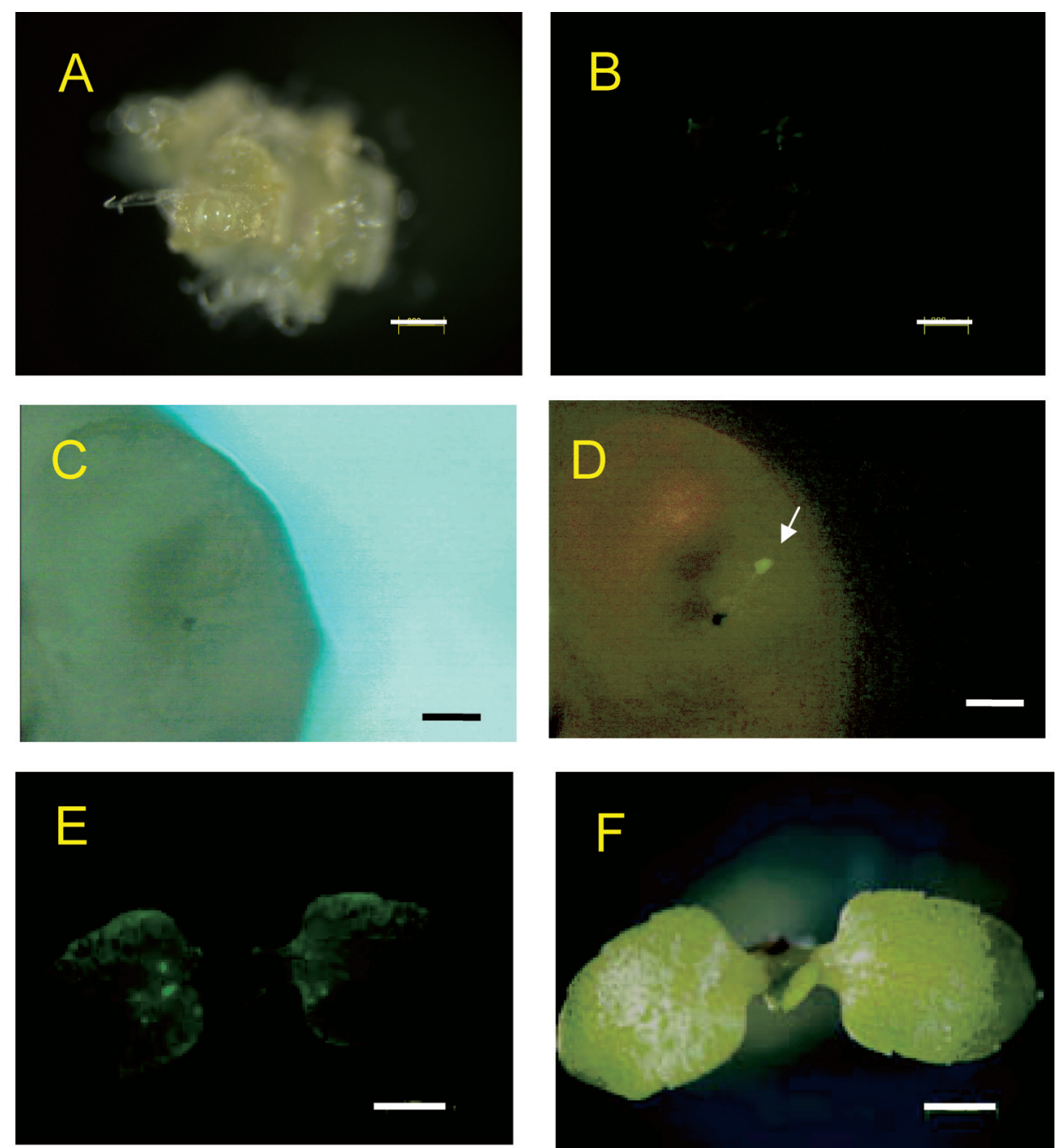

Figure 6. Micrographs of transformed Torenia apex samples. Expression of $s G F P$ was observed under the fluorescence microscope just after (A), $24 \mathrm{~h}(\mathrm{C})$ and 55 days (E) after gene introduction. (B), (D) and (F) are the bright-field images corresponding to (A), (C) and (E) respectively. The arrow in (B) indicates the single cell expressing $s G F P$. (Scale bar: $200 \mu \mathrm{m}$ in A and B, $500 \mu \mathrm{m}$ in $\mathrm{C}$ and D and $1 \mathrm{~mm}$ in $\mathrm{E}$ and $\mathrm{F}$ ) 
particle were intact (calculated based on the molecular weight of the plasmid, i.e., 9.3 MDa); according to previous reports, this amount is considered to be sufficient for transformation (Rasco-Gaunt et al. 1999). This result indicates that the laser-irradiation conditions used in this experiment had practically no harmful effect on the foreign genes.

\section{Foreign-gene introduction into Torenia by laser irradiation}

Gene introduction into Torenia tissue was performed under the irradiation condition decided based on cellviability and DNA-damage data $(3 \mathrm{~nJ} / \mathrm{shot})$. Shoot apices (277 samples) and stem segments (297 samples) were tested; 42 of the former and 85 of the latter survived. These viable samples were analyzed for their uptake of the foreign genes. All the control samples died during the selection process.

\section{Analysis of the introduced genes}

Figure 5 shows micrographs of the epidermal cells before (A) and after (B) the introduction of the genecoated gold particles by laser irradiation. The circles indicate the targeted gold particles. Although a slight trace was observed on the cell-wall surface, no major extrusion of the cell contents was observed. The treated tissue samples were observed under a fluorescence microscope and checked for $s G F P$ expression (Figure 6). Approximately $10 \%$ of the treated samples possessed cell(s) that expressed $s G F P 24 \mathrm{~h}$ after the gene introduction (Figure 6C), whereas the non-transformed samples did not exhibit fluorescence (data not shown). Furthermore, the green fluorescence was not observed around the treated cell in the immediate aftermath of gene introduction procedure (Figure 6A), indicating that the laser treatment did not cause autofluorescense of the treated region. Figure $6 \mathrm{E}$ indicates a shoot that developed on the selection medium from the gene-introduced apical cells. Fluorescent microscopic observation of the shoot clearly indicated that the transformant was chimeric. This means that target cells such as germ cells can be selected to produce a complete transgenic plant.

The uptake of foreign genes ( $S G F P$ and $H P T$ ) was further confirmed by PCR. Figure 7 indicated the example of PCR results from randomly selected 7 regenerants after gene introduction (lane 1-3: regenerants from shoot apices, lane 4-7: regenerants from stem segments). In this example, the transgenes were observed in one plantlet both in the case of $s G F P$ and HPT. Based on the trial number, the transformation efficiency was calculated to be approximately $0.4 \%$ in both the apical and epidermal samples. In biolistic transformation, the transformation efficiency is approximately $30 \%$ based on the trial number (Baum et al. 1997); however, over $10^{6}$ particles are required per trial in this method (Helios
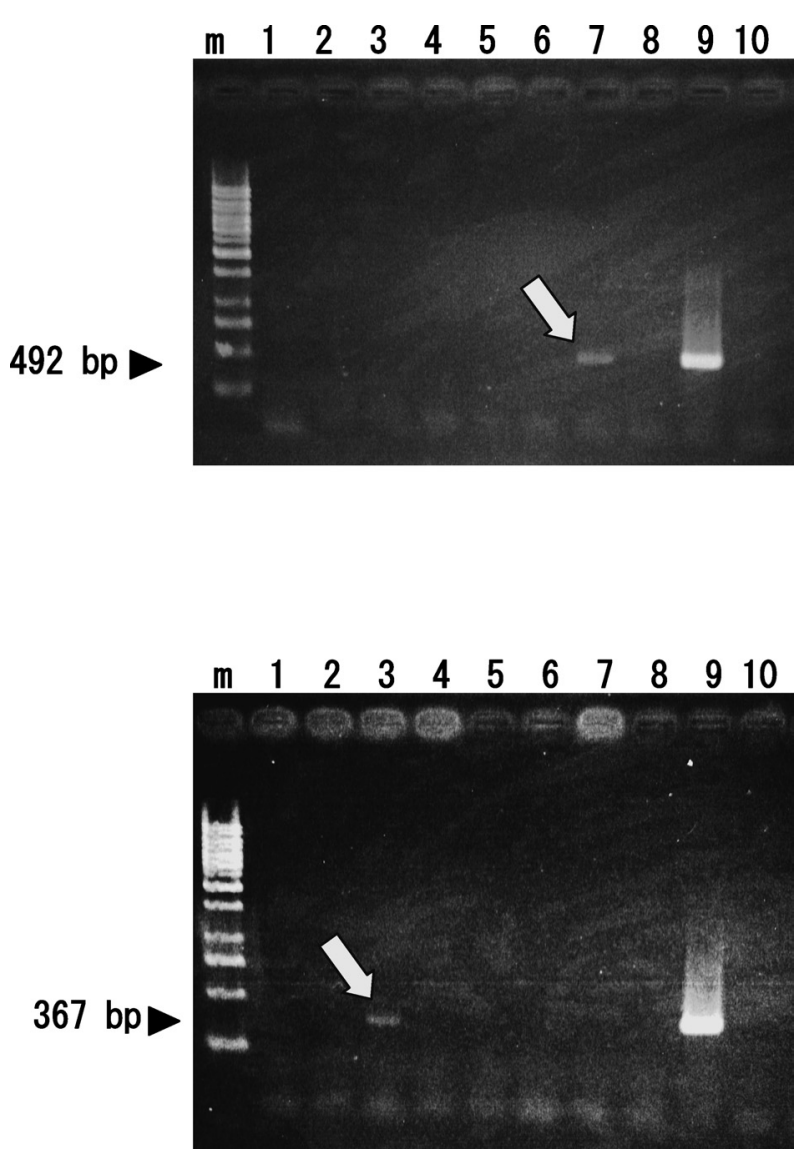

Figure 7. PCR detection of transgenes ( $S G F P(S 65 T)$ and HPT) in shoots after gene introduction. (A) 492-bp fragment of $s G F P$ ( $S 65 T$ ). (B) 367-bp fragment of HPT. m: marker; 1-3: regenerants arising from shoot apices; 4-7: regenerants arising from stem segments; 8: nontransformant (negative control); 9: pBIsGFP (S65T) (positive control); and 10: water. The white arrows indicated the positive bands.

Gene Gun manual, Bio-Rad). In contrast, our method requires only $1-10$ particles per trial. Therefore, the transformation efficiency can be considered to be even higher than that of biolistic transformation when compared in terms of the number of particles introduced. Moreover, the biolistic and Agrobacterium-mediated methods cannot be used for cell-specific transformation, e.g., in transient gene-suppression experiments using RNAi constructs. In this regard, our new method is a beneficial transformation method.

In conclusion, we have developed a new approach for the transfection of exogenous materials, e.g., foreign genes, into intact plant cells. In this method, damage to the target tissue is minimal, and foreign genes are robustly introduced only into the targeted cell. This method differs from other transfection methods in this regard. Although this method requires a suitable laser setup and is time-consuming, these drawbacks are expected to be overcome by automated equipment. Moreover, this method can possibly be used for the introduction of not only DNA but also biologically active substances such as protein regulators or inhibitors, whose 
introduction into cells is difficult through the cell wall. The use of this method for the introduction of protein compounds is currently under investigation.

\section{Acknowledgements}

The hollow optical fiber used in this experiment was supplied by Dr. Yuji Matsuura (Department of Electrical Communications, Graduate School of Engineering, Tohoku University). The plasmid was kindly provided by Dr. Norihiro Mitsukawa (Toyota Central R\&D Labs.). The authors are also grateful to Dr. Akio Okamoto (Technology Research Institute of Osaka Prefecture) who provided useful suggestions for aluminum coating the laser-condensing tip. This work was supported by the grants from the Promotion of Basic Research Activities for Innovative Biosciences program of BRAIN (Bio-oriented Technology Research Advancement Institution, Japan) and from the Grant-in-Aid for Scientific Research (KAKENHI) in Priority Area "Molecular Nano Dynamics" from Ministry of Education, Culture, Sports, Science and Technology to A.K.

\section{References}

Bock R (2001) Transgenic plastids in basic research and plant biotechnology. J Mol Biol 312: 425-438

Baum K, Groning B, Meier I (1997) Improved ballistic transient transformation conditions for tomato fruit allow identification of organ-specific contributions of I-box and G-box to the RBCS2 promoter activity. Plant J 12: 463-469

Daniell H (1997) Transformation and foreign gene expression in plants mediated by microprojectile bombardment. Methods Mol Biol 62: 463-490

Hohn B, Koukolikova-Nicola Z, Bakkeren G, Grimsley N (1989)
Agrobacterium-mediated gene transfer to monocots and dicots. Genome 31: 987-993

Kajiyama S, Shoji T, Okuda S, Izumi Y, Fukusaki E, Kobayashi A (2006) A novel microsurgery method for intact plant tissue at the single cell level using ArF excimer laser microprojection. Biotechnol Bioeng 93: 325-331

Marfori EC, Kajiyama S, Fukusaki E, Kobayashi A (2003) Phytotoxicity of the tetramic acid metabolite trichosetin. Phytochemistry 62: 715-721

Matsuura Y, Miyagi M (1999) Aluminum-coated hollow glass fibers for ArF-excimer laser light fabricated by metallorganic chemical-vapor deposition. Appl Opt 38: 2458-2462

Neuhaus G, Spangenberg G (1990) Plant transformation by microinjection techniques. Physiol Plant 79: 213-217

Niwa Y, Hirano T, Yoshimoto K, Shimizu M, Kobayashi H (1999) Non-invasive quantitative detection and applications of nontoxic, S65T-type green fluorescent protein in living plants. Plant $J$ 18: 455-463

Prescott A, Briddon R, Harwood W (1998) Plant transformation. Molecular Biomethods Handbook 251-269

Rakoczy-Trojanowska M (2002) Alternative methods of plant transformation-a short review. Cell Mol Biol Lett 7: 849-858

Rasco-Gaunt S, Riley A, Barcelo P, Lazzeri PA (1999) Analysis of particle bombardment parameters to optimize DNA delivery into wheat tissues. Plant Cell Rep 19: 118-127

Russell DR, Wallace KM, Bathe JH, Martinell BJ, McCabe DE (1993) Stable transformation of Phaseolus vulgaris via electricdischarge mediated particle acceleration. Plant Cell Rep 12: 165-169

Tanimoto S, Harada H (1984) Roles of auxin and cytokinin in organogenesis in Torenia stem segments cultured in vitro. $J$ Plant Physiol 115: 11-18

Yoshida M (2004) Generation and properties of laser-induced shock waves. Purazuma Kaku Yugo Gakkaishi 80: 427-431 (in Japanese) 